\title{
VI. Polynomiale Transformationen und Auswahlaxiom
}

von Martin Fürer

Einige der in den Vorträgen III und IV behandelten Transformationen beweisen nicht nur die polynomiale Aequivalenz von Sprachen, sondern zeigen auch (wis E. Specker bemerkt hat) die Gleichwertigkeit verschiedener Abschwächungen des Auswahlaxioms. In der axiomatischen Mengenlehre werden nämlich Aequivalenzbeweise auch mit Transformationen geführt. Ein typisches Beispiel hiefür ist (Läuchli 1971), worin folgender Satz bewiesen ist (in der Mengenlehre ohne Auswahlaxiom):

Die beiden folgenden Aussagen $A_{1}, A_{2}$ (welche beide direkt aus dem Auswahlaxiom folgen, aber das Auswahlaxiom nicht implizieren) sind äquivalent:

$A_{1}$ : Jeder nicht dreifärbbare Graph besitzt einen endlichen nicht dreifärbbaren Teilgrephen.

$A_{2}$ : Jede unerfüllbare Menge von aussagenlogischen Formeln besitzt eine endliche unerfüllbare Teilmenge.

Der Beweis der Implikation $A_{1} \Longrightarrow A_{2}$ wird bei Läuchli so geführt, dass eine Transformation angegeben wird, welche aussagenlogische Formelmengen so in Graphen transformiert, dass erfüllbare Formelmengen in färbbare Graphen übergehen und jeder endliche Teilgraph von einer endichen Formelmenge herkommt.

In diesem Vortrag wollen wir einige neue polynomiale Transformationen angeben, welche solche Sprachen direkt ineinander transformieren, welche wir bisher nur mit komplizierten Umwegen über andere Sprachen ineinander transformieren konnten. Diese direkten Transformationen bewirken unmittelbar Verkürzungen von Beweisen im 2. Kapitel. Dort führen wir Aequivalenzbeweise in der Mengenlehre ohne Auswahlaxiom mit Hilfe von polynomialen Transformationen. Wir untersuchen einige Aussagen, deren Aequivalenz von (Lośs und Ryll-Nardzewski 1955), (Mycielski 1981), (Lévy 1963) und (Läuchli 1971) gezeigt wurde. Für die Aequivalenz gewisser Aussagen können wir einfachere Beweise angeben. Damit lösen wir insbesondere ein von (Läuchli 1971) gestelltes Problem. (Wir geben einen direkten Beweis von $P_{3} \Longrightarrow P_{4}$ (VI.15)). Wir zeigen auch von einigen weiteren Aussagen, dass sie zu den übrigen äquivalent sind. 


\section{Transformationen}

Ein unterteilter Graph mit Komponenten der Grösse k ist ein Graph, dessen Punktmenge die Vereinigung von k-elementigen disjunkten Mengen ( = Komponenten) ist und dessen Kanten nur Punkte aus verschiedenen Komponenten miteinander verbinden. Ein unterteilter Graph wird wie ein gewöhnlicher Graph kodiert (III.3). Dabei wird die Unterteilung durch die Reihenfolge der Punkte in der Kodierung des Graphen festgelegt: Für alle $i$ mit $1 \leq i \leq \frac{|P|}{k}$ bildet die Punktmenge $\left\{p_{(i-1) \cdot k+1}, p_{(i-1) \cdot k+2}, \cdots, p_{i \cdot k}\right\}$ eine Komponente.

Für $k \geq 3$ ist die Sprache $k$-AUSWAHL-CLIQUE vollständig. Sie besteht aus denjenigen unterteilten Graphen mit Komponenten der Grösse $k$, welche die Eigenschaft haben, dass eine Clique (III.4) existiert, die aus jeder Komponente genau einen Punkt enthält.

Beweis ERFUELLBARKEIT MIT HOECHSTENS $k$ VARIABLEN PRO KLAUSEL $\leq$ k-AUSWAHL-CLIQUE wird gleich bewiesen wie SPEZIELLE ERFUELLBARKEIT $\leq \pi$ CLIQUE (III.4).

\section{Einige direkte Transformationen}

Bis jetzt haben wir viele polynomiale Transformationen kennengelernt: Im Beweis der Vollständigkeit von $E^{0}$ ( $E^{0}$ = Menge der Kodierungen von erfüllbaren aussagenlogischen Formeln in konjunktiver Normalform, II.5) wurde gezeigt, wie jede Sprache L aus NP (NP = Menge der nichtdeterministisch in polynomialer Zeit erkennbaren Sprachen, II.3) polynomial auf $E^{0}$ transformiert werden kann (Vortrag II). Dabei werden die möglichen Berechnungen einer nichtdeterministischen Turingmaschine beschrieben, welche diese Sprache akzeptiert. Diese Transformationen sind deshalb sehr kompliziert. Nachher wurde die Vollständigkeit von weiteren Sprachen gezeigt, indem bekannte vollständige Sprachen auf weitere Sprachen aus NP transformiert wurden. Wir können jetzt insbesondere jede als vollständig erkannte Sprache durch eine Reihe von polynomialen Transformationen mit einem Umweg über $E^{O}$ in jede andere als vollständig erkannte Sprache transformieren. Zusammensetzungen von polynomialen Transformationen sind zwar wieder polynomiale Transformationen, aber oft gibt es einfachere direkte Transformationen.

Solche direkten Transformationen können uns aus mehreren Gründen interessieren: 
- Sie helfen die Verwandtschaft einiger vollständiger Sprachen darzustellen.

- Manchmal kann der Grad von Transformationspolynomen verringert werden.

- Im nächsten Kapitel brauchen wir speziell schöne Transformationen: Teilobjekte müssen auf Teilobjekte abgebildet werden, so dess sich die Transformationen auf unendliche ubjekte erweitern lassen. Transformationen, welche einen Umweg über CLIQUE, HAMILTON-ZYKLUS Usw. machen, sowie die allgemeine Transformation nach $E^{0}$ (mit Beschreibung der Berechnungen einer nichtdeterministischen Turingmaschine) erfullen diese Forderung nicht. Ferner wollen wir zum Teil schon bekannte Sätze einfacher beweisen. Dazu brauchen wir mäglichst einfache Transformationen.

Direkte polynomiale Transformation von $2^{n}$-FAERBBARKEIT auf DREIFAERBBARKEIT

Wir kennen bereits eine polynomiale Transformation von $2^{n}$-FAERBBARKEIT über $E^{\circ}$ und ERFUELLBARKEIT MIT HOECHSTENS DREI VARIABLEN PRO KLAUSEL nach DREIFAERBBARKEIT. Wir wollen hier eine viel einfachere Transformation behandeln. Diese direkte Transformation liefert uns sehr einfache Transformationen von k-FAERBBARKEIT nach $\ell$-FAERBBARKEIT für beliebige $k$ und $\&$ mit $\ell \geq 3$, denn fur $k \leq l$ ist diese Transformation trivial $(I V, 3)$.

Einem Grephen $G=(P, K)$ wird ein Graph $G^{\prime}=\left(P^{\prime}, K^{\prime}\right)$ zugeordnet, so dass $G 2^{n}$-färbbar $\Longrightarrow G^{\prime} 3$-färbbar.

Die Menge der Punkte $P$ sei geordnet

G:

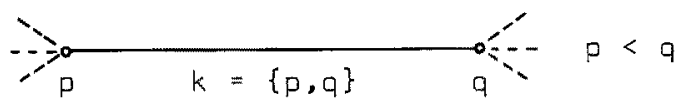


$\sigma^{*}:$

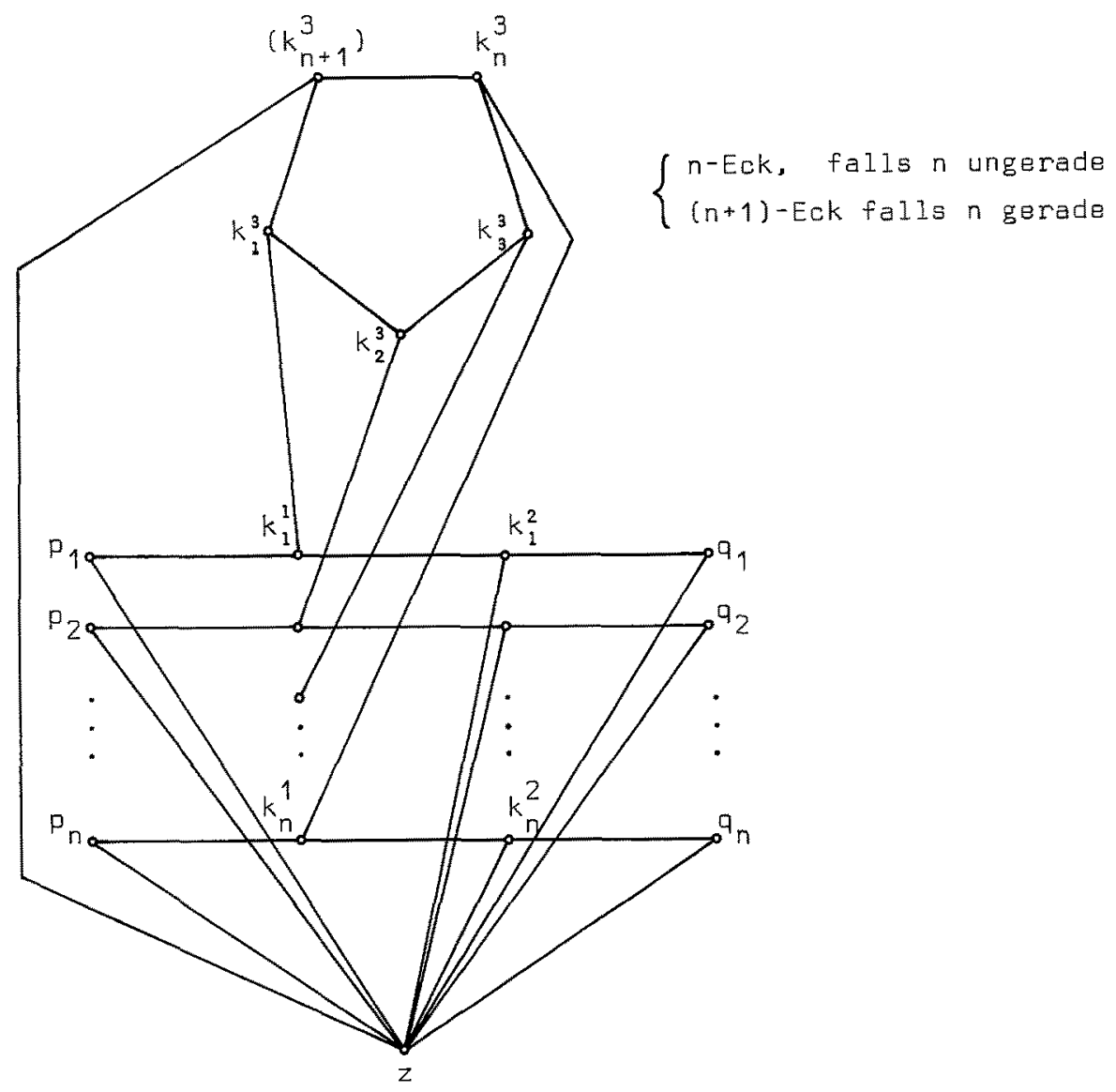

$G^{\prime}=\left(P^{\prime}, K^{\prime}\right) \mathrm{mit}$

$$
\begin{gathered}
P^{\prime}=\{z\} \cup\left\{p_{i} \mid 1 \leq i \leq n, p \in P\right\} \cup\left\{k_{i}^{j} \mid 1 \leq i \leq n, j \leq j \leq 3, k \in k\right\} \\
\text { und falls } n \text { gerade } \cup\left\{k_{n+1}^{3} ; k \in k\right\} .
\end{gathered}
$$

Jedem Punkt $p$ von $G$ werden also $n$ Punkte und jeder Kante $k$ von $G$ werden $3 n$ (bzw. $3 n+1$ falls $n$ gerade) Punkte von $G^{\prime}$ zugeordnet. Hingegen ist $z$ ein einziger Zusatzpunkt zum ganzen Graphen $G^{\prime}$.

$K^{\prime}$ : siehe Zeichnung.

Falls $n$ gerade ist und deshalb Punkte $k_{n+1}^{3}$ vorkommen, so werden diese mit $z$ verbunden.

Behauptung $G$ ist $2^{n}$-färbbar $\Longleftrightarrow G$ ' ist 3 -färbbar.

Beweis Es seien $\{0,1\}^{n}$ die Farben von $G$

$$
\{0,1,2\} \text { die Farben von } G^{\prime} \text {. }
$$


$\Longrightarrow f$ sei eine $2^{n}-F a ̈ r b u n g ~ v o n ~ G . ~\{p, q\}$ sei eine beliebige Kante von $G$. $p$ habe die Farbe $\left(s_{1}, \ldots, s_{n}\right)$, q habe die Farbe $\left(t_{1}, \ldots, t_{n}\right)$ mit $s_{m} \neq t_{m}$ für ein $m$.

Wir ergänzen $f^{\prime}\left(p_{i}\right)=s_{i}, f^{\prime}\left(q_{i}\right)=t_{i}, f^{\prime}(z)=2$ zu einer Färbung derjenigen Punkte von $G^{\prime}$, welche der Kante $\{p, q\}$ zugeordnet sind: $f^{\prime}\left(k_{i}^{1}\right)= \begin{cases}2 \text { für } i \neq m & \text { (Es dürfen nicht alle } k_{i}^{1} \text { mit } 2 \text { gefärbt } \\ t_{m} \text { für } i=m & \text { werden, sonst ist das ungerade Vieleck } \\ & \text { nicht mehr färbbar). }\end{cases}$ $f^{\prime}\left(k_{i}^{2}\right)=1-t_{i}$ $f^{\prime}\left(k_{m}^{3}\right)=2$ $k_{i}^{3}$ für $i \neq m$ färbt man abwechslungsweise mit 0 und 1 .

=Sei $f^{\prime}$ eine Färbung von G'. G.B.d.A. nehmen wir an $f^{\prime}(z)=2$. $z u$ jeder Kante $(p, q)=k$ von $G$ gibt es ein $m \neq n+1$ mit $f^{\prime}\left(k_{m}^{3}\right)=2$ (ungerades Vieleck).

Also ist $f^{\prime}\left(k_{m}^{1}\right) \neq 2$ und damit $f^{\prime}\left(p_{m}\right) \neq f^{\prime}\left(q_{m}\right)$.

Für alle Punkte $p$ von $G$ kann man also die Färbung $f(p)=\left(f^{\prime}\left(p_{1}\right), \ldots, f^{\prime}\left(p_{n}\right)\right)$ wählen. Dann gilt $f(p) \neq f(q)$ für jede Kante $\{p, q\}$ von $G$.

\section{Bemerkungen}

1. Mit einer ähnlichen Methode kann $n^{2}$-FAERBBARKEIT direkt auf $(n+1)$-FAERBBARKEIT transformiert werden.

Der Fall $2^{n}=n^{2}=4, n+1=3$ wird dann noch etwas einfacher:

G:
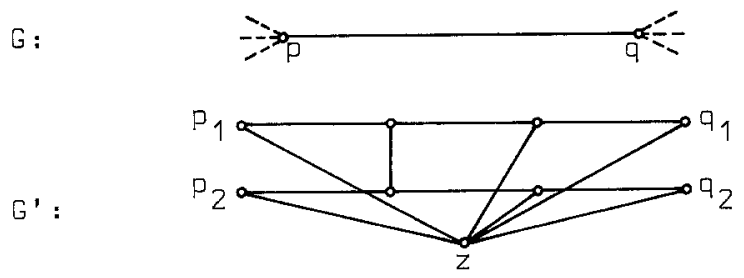

2. Auch die $n^{m}$-FAERBBARKEIT ist mit dieser Methode in einem Schritt auf die $(n+1)$-FAERBBARKEIT transformierbar.

Direkte polynomiale Transformation von $E^{\circ}$ auf DREIFAERBBARKEIT

Wir kennen schon eine salche Transformation, welche einen Umweg macht über ERFUELLBARKEIT MIT HOECHSTENS 3 VARIABLEN PRO KLAUSEL. Eine direkte Transformation von $E^{\circ}$ auf Dreifärbbarkeit liefert uns die Methade, 
mit der wir $2^{n}$-FAERBBARKEIT auf DREIFAERBBARKEIT transformiert haben. Sei $F$ die aussagenlogische Formel $c_{1} \wedge \ldots \wedge c_{m}$ mit $c_{i}=y_{i 1} \vee_{\ldots} \ldots v_{i n_{i}}$, wobei $y_{i j} \in\left\{x_{1}, \ldots, x_{n}, \bar{x}_{1}, \ldots, \bar{x}_{n}\right\}$. Der Formel $F$ wir der folgende Graph $G=(P, K)$ zugeordnet:

$$
\begin{aligned}
P= & \{p, z\} \cup\left\{x_{i} \mid 1 \leq i \leq n\right\} \cup\left\{\bar{x}_{i} \mid 1 \leq i \leq n\right\} \\
& \cup\left\{p_{i j}^{k} \mid 1 \leq i \leq m, 1 \leq j \leq n_{i}, k \in\{1,3\}\right\} \\
& \left.\cup p_{i, n_{i}+1}^{3} \mid 1 \leq i \leq m, n_{i} \text { gerade }\right\}
\end{aligned}
$$

$K:$ Für alle $i$ mit $1 \leq i \leq n$ haben wir die Kanten:

$\left\{x_{i}, \bar{x}_{i}\right\},\left\{z, x_{i}\right\},\left\{z, \bar{x}_{i}\right\}$,

sowie für alle $i$ mit $1 \leq i \leq m$ und für alle $j$ mit $1 \leq j \leq n_{i}$ die Kanten:

$\left\{p_{i j}^{1}, y_{i j}\right\},\left\{p, p_{i j}^{1}\right\},\left\{p_{i j}^{1}, p_{i j}^{3}\right\}$.

(Auch beim Graphen verwenden wir $y_{i j}$ als Namen für den entsprechenden Punkt $x_{k}$ bzw. $\bar{x}_{k}$.

Weiter haben wir die Kante $\{p, z\}$, und für jedes feste $i$ sind die Punkte $P_{i j}^{3}$ zu einem ungeraden Vieleck verbunden.

Falls $n_{i}$ gerade ist, und deshalb ein Punkt $p_{i, n_{i}+1}^{3}$ vorkommt, so ist dieser mit z verbunden.

Beispiel $\left(\bar{x}_{1} \vee x_{2} \vee \bar{x}_{4}\right) \wedge\left(x_{1} \vee \bar{x}_{2} \vee x_{3} \vee x_{4}\right)$

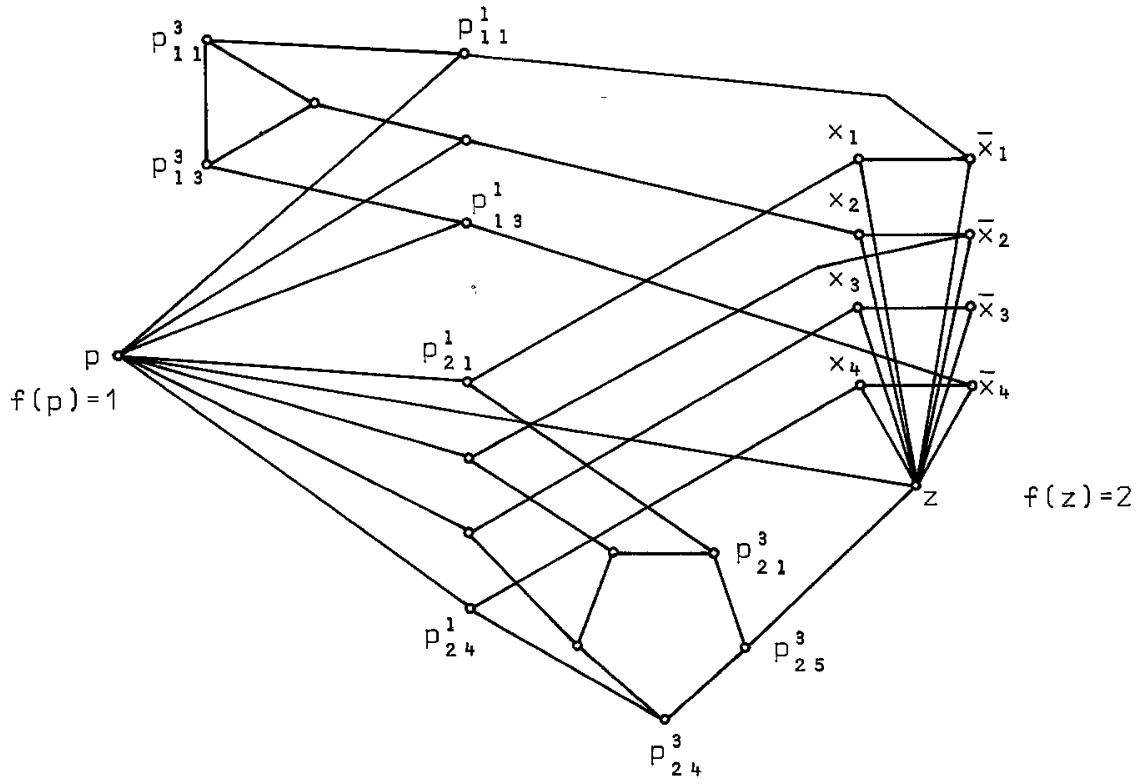

Behauptung $F$ ist erfüllbar $\Longrightarrow G$ ist 3 -färbbar. 
Beweis Wir färben $G$ mit $\{0,1,2\}$. 0.B.d.A. habe $p$ die Farbe 1 und $z$ die Farbe 2.

$\Longrightarrow A$ sei erfüllbar. Es gibt also eine Belegung $v($ II.5) mit $v(F)=1$. Dann gibt es in jeder Klausel $C_{i}$ ein kleinstes $j_{0}$ mit $v\left(y_{i j o}\right)=1$. Wir können deshalb leicht eine Färbung $f$ des Graphen angeben mit $f\left(y_{i j}\right)=v\left(y_{i j}\right)$. Dazu wählen wir insbesondere $f\left(p_{i j}^{1}\right)=2$ für $j \neq j_{0}$, $f\left(p_{i j_{0}}^{1}\right)=0$ und $f\left(p_{i j_{0}}^{3}\right)=2$.

$\Longrightarrow$ Jede Färbung $f: P \rightarrow\{0,1,2\}$ mit $f(z)=2$ und $f(p)=1$ definiert eine Variablenbelegung $v$ durch $v\left(x_{i}\right)=f\left(x_{i}\right)$. Zu jeder Klausel $c_{i}$ gibt es ein $j_{0}$ mit $f\left(x_{i j}\right)=f(p)=1$, also gilt $v\left(y_{i j_{0}}\right)=f\left(y_{i j_{0}}\right)=1$ und
damit $v(F)=1$.

Direkte polynomiale Transformation von $k$-AUSWAHL-CLIQUE auf $k$-FAERBBARKEIT für $k \geq 3$

Diese Transformation brauchen wir im nächsten Kapitel.

Es sei $G=(P, K)$ ein unterteilter Graph mit Komponenten der Grösse $k$. Dann ist $P=\bigcup_{i=1}^{m} P^{i}$ für die Komponenten $P^{i}=\left\{p_{1}^{i}, \ldots p_{k}^{i}\right\}$. In $G$ sind nur Punkte aus verschiedenen Komponenten durch Kanten verbunden.

Dem unterteilten Graphen $G$ wird ein Graph $G^{\prime}=\left(P^{\prime}, K^{\prime}\right)$ zugeordnet durch $P^{\prime}=P \cup\left\{p_{0}\right\} \cup\left\{(p, q, l) \mid(\exists i, j) 1 \leq i<j \leq m, p \in p^{i}, q \in p^{j},\{p, q\} \notin k\right.$ und $\left.1 \leq l \leq k\right\}$

$K^{\prime}=\left\{\{p, q\} \mid(\exists i) p \in p^{i}\right.$ und $\left.q \in p^{i}\right\} \cup\left\{\left\{(p, q, l),\left(p, q, l^{\prime}\right)\right\} \mid(p, q, l) \in p^{\prime}\right.$, $\left(p, q, \ell^{\prime}\right) \in p^{\prime}$ und $\left.\ell \neq \ell^{\prime}\right\}$

$U\left\{\left\{p_{0},(p, q, l)\right\} \mid(p, q, l) \in P^{\prime}\right.$ und $\left.3 \leq l \leq k\right\}$

$U\left\{\{p,(p, q, 1)\} \mid(p, q, 1) \in P^{\prime}\right\}$

$U\left\{\{q,(p, q, 2)\} \mid(p, q, 2) \in P^{\prime}\right\}$

Wir nehmen also den komplementären Graphen $\left(P,\left(l_{2}^{P}\right) K\right)$ von $G$, wählen einen zusätzlichen Punkt $P_{0}$ und ersetzen jede Kante $\{p, q\}$ zwischen zwei verschiedenen Komponenten durch:

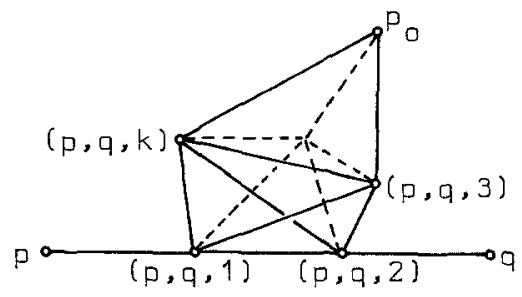




\section{Beispiel}

G :

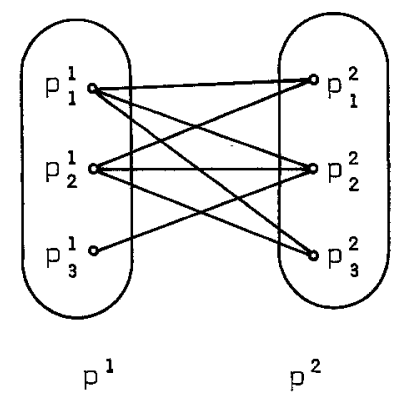

G':

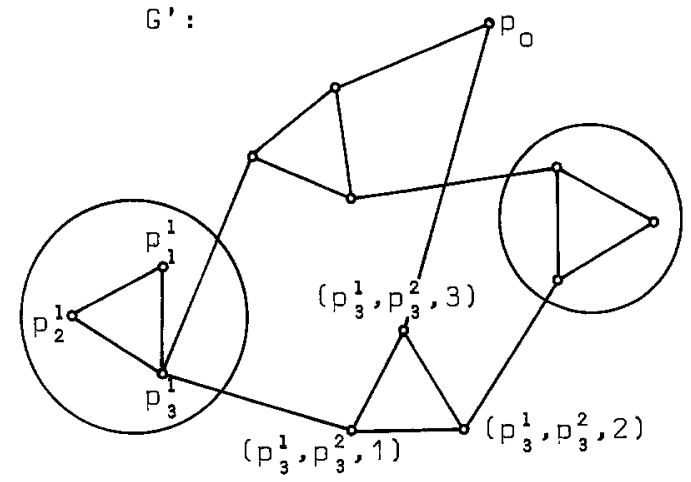

Behauptung Für $k \geq 3$ gilt:

G besitzt eine k-Auswahl-Clique $\Longleftrightarrow G^{\prime}$ ist k-färbbar.

\section{Beweis}

$\Longrightarrow P_{0}$ und alle Punkte der k-Auswahl-Clique werden mit 0 gefärbt. Für die restlichen $k-1$ Punkte jeder Komponente werden alle übrigen Farben verwendet.

Falis $p, q \in P$ und $\{p, q\} \notin k$, so färben wir $(p, q, 1),(p, q, 2), \ldots,(p, q, k)$ der Reihe nach mit möglichst kleinen Farben. Weil $p$ und $q$ nicht beide mit 0 gefärbt sind, so bekommt $(p, q, 1)$ oder $(p, q, 2)$ die Farbe 0 . Also wird $f\left((p, q, \ell) \neq 0=f\left(p_{0}\right)\right.$ für $3 \leq \ell \leq k$.

$\Longleftarrow$ In jeder Komponente von $P$ gibt es genau einen Punkt mit der gleichen Farbe wie $P_{0}$. Diese Punkte bilden eine k-Auswahl-Clique in $G$. Wären nämlich zwei solche Punkte $p$ und $q$ in $G$ durch keine Kante verbunden, so wäre in $G^{\prime}$ von den $k$ Punkten $\{(p, q, l) \mid 1 \leq l \leq k\}$ keiner mit $f\left(p_{0}\right)$ gefärbt.

Bemerkung Die umgekehrte Transformation von k-FAERBBARKEIT auf k-AUSWAHL-CLIQUE ist trivial.

Direkte polynomiale Transformation von k-AUSWAHL-CLIQUE auf 3-FAERBBARKEIT für $k \geq 1$

Diese Transformation erhalten wir durch eine kleine Aenderung der Transformation von k-AUSWAHL-CLIQUE auf $k$-FAERBBARKEIT: $\ell \leq k$ ersetzen wir durch $\ell \leq 3$, und statt alle Punkte einer Komponente miteinander $z u$ verbinden, machen wir daraus nur ein Vieleck mit ungerader Eckenzahl. 
Falls $k$ gerade ist, so wählen wir dazu in jeder Komponente einen Zusatzpunkt, der mit $p_{0}$ verbunden wird.

Direkte polynomiale Transformation von $(m \cdot n)$-AUSWAHL-CLIQUE nach $(m+n-1)-A U S W A H L-C L I Q U E$

Der $i$-ten Komponente $P^{i}=\left\{p_{1}^{i}, \ldots, p_{m}^{i} n_{i j}\right.$ ordnen wir n Komponenten $Q^{i j}=\left\{p^{i}(j-1) \cdot m+1, \ldots, p_{j}^{i} \cdot m, q_{1}^{i j}, \ldots, q_{n-1}^{i j}\right\}$ mit $1 \leq j \leq n z u$. Die früher schon vorhandenen Punkte ( $p$-Punkte) werden untereinander gleich verbunden wie vorher, während $q_{l}^{i j}$ mit allen Punkten der anderen Komponenten

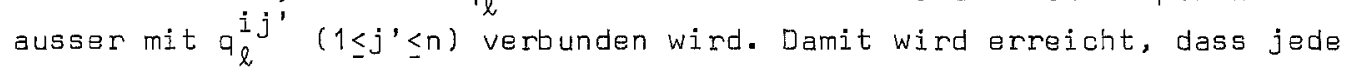
$(m+n-1)$-Auswahl-Clique für alle i genau einen p-Punkt auswählt.

\section{Mengenlehre ohne Auswahlaxiom}

Auswahlaxiom Zu jeder Familie F von nichtleeren Mengen gibt es eine Funktion $f$ mit $f(M) \in M$ für jede Menge M aus der Familie F.

Eine Abschwächung des Auswahlaxioms ist ein Satz, der aus dem Auswahlaxiom folgt, aber das Auswahlaxiom nicht impliziert und auch nicht ohne Auswahlaxiom beweisbar ist. Viele bekannte Abschwächungen des Auswahlaxioms sind zum Kompaktheitssatz für die Aussagenlogik äquivalent, und ihre Aequivalenz wurde oft über diesen Satz bewiesen. (Genau genommen wurden diese Aequivalenzen über den gleichwertigen Booleschen Primidealsatz bewiesen, welcher aber oft kompliziertere Transformationen erfordert).

Kompaktheitssatz für die Aussagenlogik Sei M eine unendliche Menge von aussagenlogischen Formeln. (Auch die Variablenmenge darf eine beliebige Mächtigkeit haben). Falls jede endliche Teilmenge von M erfüllbar ist, so ist auch Merfüllbar.

Zu einigen Aussagen, welche von einer anschaulichen kombinatorischen Art sind, können wir aber mit direkten polynomialen Transformationen viel einfachere Aequivalenzbeweise führen. Dabei ist es für die Richtigkeit der Beweise natürlich unwesentlich, dass diese Transformationen, welche wir aus der Komplexitätstheorie übernehmen, polynomial sind. 
Satz In der Mengenlehre ohne Auswahlaxiom sind die folgenden Aussagen äquivalent:

(1) I: Eine unendliche Menge von aussagenlogischen Formeln ist genau dann erfülbar, wenn jede endliche Teilmenge erfüllbar ist.

(2) Zu einem unendlichen System $\left\{U_{i} \mid i \in I\right\}$ von endlichen Teilmengen $U_{i}$ einer Menge $N$ gibt es genau dann eine Schnittmenge $U$ (d.h. Un $U_{i}=$ einelementige Menge für alle $i \in I)$, wenn es zu jedem endlichen Teilsystem $\left.\left\{U_{j}\right\} j \in J\right\}$ ( $J$ endliche Teilmenge von $I$ ) eine Schnittmenge gibt.

(3) Die $S_{i}$ mit $i \in I$ seien Teilmengen einer unendlichen Menge M mit $\left\{i \mid a \in S_{i}\right\}$ endlich für alle a $\in$ M.

Es gibt eine Partition von M (d.h. es gibt JC I mit disjunkten $s_{j}$ für $j \in J$ und $\underset{j \in J}{\bigcup} S_{j}=M$ ) genau dann, wenn es zu jeder endlichen Teilmenge $M$ ' von M eine Partition gibt (d.h. es gibt JCI mit $S_{j} \cap M$ ' disjunkt für alle $j \in J$ und $\left.\bigcup_{j \in J}\left(S_{j} \cap M^{\prime}\right)=M^{\prime}\right)$.

(4) $P_{k}$ : Ein unendlicher Graph $G$ ist genau dann $k$-färbbar $(k \geq 3)$, wenn jeder endliche Teilgraph k-färbbar ist.

(5) $S_{k}$ : In einem unendichen unterteilten Graphen mit Komponenten der Grösse $k$ ( $k \geq 3$ endlich) gibt es genau dann eine k-Auswahl-Clique (VI.2) ( = Clique mit einem Punkt aus jeder Komponente), wenn es in jedem Teilgraphen, der aus endlich vielen Komponenten und den dazwischen liegenden Kanten gebildet wird, eine k-Auswahl-Clique gibt.

(6) Eine unendliche Menge von aussagenlogischen Formeln in konjunktiver Normalform mit höchstens $k(k \geq 3)$ Variablen pro klausel ist genau dann erfülbar, wenn jede endliche Teilmenge erfüllbar ist.

\section{Bemerkungen}

1. Die Aussagen $I, P_{k}$ und $S_{k}$ wurden schon früher untersucht. Die Aussagen (2) und (3) hat E. Specker vorgeschlagen.

Die Implikationen $S_{4} \Longrightarrow I, I \Longrightarrow S_{m}$ für alle $m$ und $s_{m} \Rightarrow s_{k}$ für $m \geq k$ stammen von (Kośs und Ryll-Nardzewski 1955), $S_{n} \Longrightarrow P_{n}$ und $P_{n+1} \Longrightarrow P_{n}$ für $n \geq 2$ von (Mycielski 1961), $s_{3} \Longrightarrow I$ von (Lévy 1963) und $P_{3} \Longrightarrow I$ von (Läuchli 1971).

2. Der direkte Beweis von Läuchli tst recht schwierig. Auf einem Umweg kommen wir schneller zum Ziel. Wir beweisen $P_{3} \Longrightarrow S_{3}$, denn Lévy gibt einen einfachen Beweis für $\mathrm{S}_{3} \Longrightarrow I$. Trotzdem ist der Beweis von Läuchli sehr interessant, denn es wird dabei der folgende Satz über die Erweite- 
rung von Färbungen bewiesen:

Für alle $n \geq 3$, für alle endlichen Punktmengen $P^{\prime}$ und für alle Mengen $M$ von Einteilungen der Punkte $P^{\prime}$ in höchstens $n$ Klassen gibt es einen Graphen $G=(P, K)$ mit $P^{\prime} \subset P$, so dass sich genau diejenigen nuFärbungen von $P^{\prime} z u$ Färbungen des Graphen $G$ erweitern lassen, für welche die Klassen gleichfarbiger Punkte auf $P^{\prime}$ eine Klasseneinteilung aus $M$ bewirken.

Dieser Satz zeigt eine Verwandtschaft von färbbaren Graphen mit erfülbaren aussagenlogischen Formeln in konjunktiver Normalform. Für aussagenlogische formeln gilt nämlich der folgende einfache Satz:

Für jede endliche Menge von Variablen $V^{\prime}$ und jede Menge $M$ von Belegungen $v: V^{\prime} \rightarrow\{0,1\}$ gibt es eine aussagenlogische Formel $A$ in konjunktiver Normalform in den Variablen $V$ mit $V^{\prime} \subset V$, so dass genau die Variablenbelegungen von $V$ ' aus $M$ so zu Variablenbelegungen von $V$ erweitert werden können, dass dabei die Formel A erfüllt wird.

3. Die Aequivalenz dieser sechs Aussagen ist deshalb richtig, weil bei einer Transformation, d.h. einer Uebersetzung eines Problems von einer Sprache in eine andere, nicht auf das ganze unendliche System geachtet werden muss. Es müssen nur elementare endliche Bausteine übersetzt werden. Z.B. bei der Uebersetzung einer Fürbbarkeitsaussage in die Aussagenlogik werden einfach jedem Punkt und jeder Kante des Graphen je eine aussagenlogische Formel zugeordnet. Endlichen Teilsystemen in einer Sprache entsprechen deshalb endliche Teilsysteme in der anderen Sprache.

4. Die Bedingungen in (2), dass die $U_{i}$ endlich sein müssen und in (3), dass jedes Element von $M$ nur in endich vielen $S_{i}$ vorkommen darf, sind wesentlich. Ohne diese Bedingungen wären die Aussagen (2) und (3) falsch: Wir wählen $z . B$. in (3) als $S_{i}$ alle endlichen Teilmengen von $M$, welche ein fest gewähltes Element a $\in$ M enthalten. Dann können wir jede endliche Teilmenge mit einem $S_{i}$ überdecken, aber M lässt sich nicht disjunkt überdecken. Für die Aussage (2) können wir leicht ein entsprechendes Beispiel finden.

5. Unter den unendilichen systemen in (1), ..., (6) soliten wir uns nicht nur abzählbare systeme vorstellen. Beschränken wir uns nämlich auf abzählbare Systeme (abzählbare Graphen usw), so lassen sich nach einer Bemerkung von Ryll-Nardzewski die Aussagen (1),...,(6) schon ohne Auswahlaxiam beweisen. 
Beweis Die eine Richtung ist trivialerweise immer richtig:

Falls ein unendlicher Graph k-färbbar ist, so ist jeder endliche Teilgraph k-färbbar, usw.

\section{$(1) \Longrightarrow(2)$}

SCHNITTMENGE (IV.5) lässt sich (polynomial) auf $E^{0}$ transformieren. Wir betrachten jetzt nur noch die Transformationen von Systemen $\left(N,\left\{U_{1}, \ldots, U_{t}\right\}\right)$ in aussagenlogische Formeln und nicht die Transformationen van Kodierungen von solchen Systemen in Kodierungen von Formeln. Solche Transformationen lassen sich in offensichtlicher Weise auf unendliche Systeme $\left(N,\left\{U_{i} \mid i \in I\right\}\right)$ erweitern. Die Menge der Variablen kann dabei überabzählbar sein, deshalb können wir im allgemeinen nicht mehr natürliche Zahlen als Indizes verwenden. Wir ordnen daher einfach jedem Element a der Menge $N$ eine Variable $x_{a}$ zu. Einer Teilmenge $u_{i}=\left\{a_{1}, \ldots, a_{n}\right\}$ wird dann die aussagenlogische Formel $\left(x_{a_{1}} \vee \ldots \vee x_{a_{n}}\right) \wedge \bigwedge_{i \leq i<j \leq n}\left(\neg x_{a_{i}} \vee \neg x_{a_{j}}\right)$ zugeordnet. ( $x_{a}$ wahr soll bedeuten: $a \in U)$.

Einer unendlichen Familie von Teilmengen $U_{i}(i \in I)$ wird so eine unendliche Formelmenge $F$ zugeordnet. (Die Disjunktionen werden nur endlich lang, weil alle $U_{i}$ endlich sind).

Damit wird das Auswahlaxiom nicht brauchen, um bei jeder Menge $U_{i}$ eine spezielle Reihenfolge ihrer Elemente $a_{1}, \ldots a_{n}$ auszuwählen, ordnen wir der Menge $U_{i}$ nicht nur eine Formel zu, sondern alle $n$ : Formeln, welche den Permutationen von $a_{1}, \ldots, a_{n}$ entsprechen.

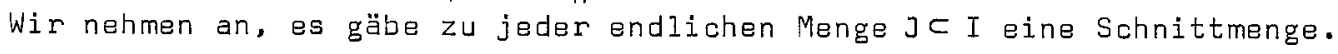
Da jede endliche Teilmenge von $F$ von endlich vielen $U_{j}(j \in J)$ herkommt, so ist sie erfüllbar. Nach (1) ist dann ganz F erfüllbar. Eine Variablenbelegung $v$, welche $F$ erfüllt, definiert aber eine Schnittmenge $U$ durch $a \in U \Longleftrightarrow v\left(x_{a}\right)=1$.

\section{$\underline{(2) \Longrightarrow(3)}$}

PARTITION $\leq_{\pi}$ SCHNITTMENGE wurde gezeigt (IV.5). Unendlich grossen Teilmengen $S_{i}$ aus PARTITION entsprechen unendlich viele Teilmengen $U_{j}$ aus SCHNITTMENGE. Alle $U_{j}$ sind endlich, weil die Durchschnitte von unendlich vielen $S_{i}$ leer sind.

Wir nehmen an, wir hätten eine unendliche Menge $M$ und ein System von Teilmengen $S_{i}$ gegeben (mit $\left\{i \mid a \in S_{i}\right\}$ endlich für alle $a \in M$ ), und $z u$ jeder endlichen Teilmenge $M$ ' von $M$ gäbe es eine Partition. Wir wollen mit Hilfe von (2) zeigen, dass es dann auch zu M eine Partition gibt. Die Transformation von PARTITION nach SCHNITTMENGE ordnet dem system 
$\left(M,\left\{S_{i} \mid i \in I\right\}\right)$ ein System $\left(N,\left\{U_{j} \mid j \in J\right\}\right)$ zu. Dabei ist $M$ genau dann Vereinigung von einigen disjunkten $S_{i}$, wenn in $\left(N,\left\{U_{j} \mid j \in J\right\}\right)$ eine Schnittmenge existiert. Die Transformation von PARTITION nach SCHNITTMENGE ordnet aber auch jedem endlichen Teilsystem (M', $\left.\left\{S_{i}^{\prime} \mid i \in I\right\}\right)$ (M' endlich) ein endliches Teilsystem ( $N,\left\{U_{j} \mid j \in J J^{\prime}\right\}$ ) (J' endlich) zu. Dabei tritt sogar jedes endliche Teilsystem von $\left(N,\left\{U_{j} \mid j \in J\right\}\right)$ als Bild eines Teilsystems von (M, $\left.\left\{S_{i} \mid i \in I\right\}\right)$ auf. Weil zu jeder endlichen Teilmenge van M eine Partition existiert, so gibt es jetzt auch zu jedem endichen Teilsystem von $\left(N_{,}\left\{U_{j} \mid j \in J\right\}\right)$ eine Schnittmenge. Nach (2) gibt es auch im ganzen System eine Schnittmenge $U$. Diese definiert aber eine Partition von $M$, denn $M$ ist disjunkte Vereinigung der $s_{j} \operatorname{mit} s_{j} \in U$.

$\stackrel{(3) \Longrightarrow(4)}{\longrightarrow}$

$k$-FAERBBARKEIT $\leq$ PARTITION zeigt man für $k \geq 3$ genau gleich wie für $k=3$ (IV.5). Ist ein Graph unendlich, so werden ihm unendlich viele (eventuell unendliche) $S_{i}$ zugeordnet, aber jedes Element von $M$ kommt nur in endlich vielen $S_{i}$ vor.

$G$ sei ein unendlicher Graph von dem jeder endliche Teilgraph k-färbbar ist. Dem Graphen $G$ wird ein unendliches System $\left(M,\left\{S_{i}: i \in I\right\}\right)$ und $j e d e m$ endlichen Teilgraphen von $G$ ein überdeckbares (d.h. es existiert eine Partition) endliches Teilsystem zugeordnet. Weil nun jedes endiche Teilsystem von ( $\left.M,\left\{S_{i}: i \in I\right\}\right)$ enthalten ist in einem Teilsystem, das einem endichen Teilgraphen von $G$ zugeordnet wird, so ist es auch überdeckbar. Nach (3) ist dann ganz M disjunkt überdeckbar. Eine Ueberdeckung von M definiert aber ein k-Färbung von $G$.

$(4) \Longrightarrow(5)$

Im ersten Kapitel (VI.7) haben wir gezeigt, dass es eine Transformation von k-AUSWAHL-CLIQUE nach k-FAERBBARKEIT gibt. Diese Transformation lässt sich sofort auf unendliche unterteilte Graphen erweitern. Dabei gibt es allerdings eine kleine Schwierigkeit, weil die kamponenten im allgemeinen nicht geordnet sind. Diese Schwierigkeit können wir überwinden, indem wir $z . B$. jedem Punktepaar $\{p, q\}$, das im unterteilten Graphen $G$ durch keine Kante verbunden ist, nicht nur den Graphen mit den Punkten $\{(p, q, l) \mid 1 \leq l \leq k\}$ zuordnen, sondern zusätzlich noch einen isomorphen Graphen mit den Punkten $\{(q, p, l) \mid 1 \leq l \leq k\}$. Wird diese Transformation jetzt auf unendliche unterteilte Graphen angewendet, so wird endlich vielen Komponenten von $G$ ein endlicher Teilgraph von G' zugeordnet, und jeder endliche Teilgraph von $G$ ' ist enthalten im Bild von endlich vielen Komponenten von G. Weil es zu je endlich vielen Komponen- 
ten von $G$ eine k-Auswahl-Clique gibt, so ist jeder endliche Teilgraph von G' k-färbbar. Damit ist nach (4) der ganze Graph G' k-färbbar. Jede $k-F \ddot{r} r b u n g$ von $G^{\prime}$ definiert aber eine k-Auswahl-Clique von $G$.

(i) $\Longrightarrow(i+1)$

Jetzt ist auch das allgemeine Schema dieser Beweise erkennbar: Die i-te Aussage handelt von bestimmten objekten (z.B. Graphen). Zu jedem Objekt gibt es eine Menge von Teilobjekten (z.B. alle Teilgraphen). Gewisse Objekte sind ausgezeichnet (z.B. die k-färbbaren Graphen). Falls ein Objekt ausgezeichnet ist, so sind auch alle seine endlichen Teilobjekte ausgezeichnet. Die Umkehrung davon wird in der i-ten Aussage behauptet. Weiter kennen wir eine Transformation. Das ist eine Abbildung $f$ : \{0bjekte aus $(i+1)\} \rightarrow$ \{0bjekte aus $(i)\}$ mit folgenden Eigenschaften: 1. endliche Teilobjekte gehen in endliche Teilobjekte.

2. Ein objekt aus $(i+1)$ ist genau dann ausgezeichnet, wenn auch sein Bild ausgezeichnet ist.

3. Jedes endliche Teilobjekt aus (i) ist enthalten im Bild eines endlichen Teilobjektes aus $(i+1)$.

Die bekannten polynomialen Transformationen zwischen den sechs obigen Problemen lassen sich in offensichtlicher Weise auf unendliche objekte ausdehnen.

Dazu sind allerdings manchmal gewisse Symmetrisierungen nötig (vgl. (1) $\Longrightarrow(2),(4) \Longrightarrow(5))$, um das Auswahlaxiom zu vermeiden. Wir können dann in jedem Falle leicht nachprüfen, dass die Bedingungen 1 bis 3 erfüllt sind. (In diesem Zusammenhang ist es natürlich ganz unwichtig, dass diese Transformationen nur polynomiale Zeit brauchen).

Zum Beweis von $(i) \Longrightarrow(i+1)$ wählen wir nun ein unendliches abjekt $0_{i+1}$ aus $(i+1)$ mit der Eigenschaft, dass jedes endliche Teilobjekt von $0_{i+1}$ ausgezeichnet ist. Wir haben dann zu zeigen, dass auch $0_{i+1}$ selber ausgezeichnet ist:

Es sei $f$ eine Transformation, welche den objekten aus ( $i+1$ ) objekte aus (i) zuordnet. Dann ist das Bild jedes endlichen Teilobjekts von $0_{i+1}$ ausgezeichnet, und da jedes endliche Teilobjekt von $f\left(0_{i+1}\right)$ enthalten ist in einem solchen Bild, so ist es auch ausgezeichnet. Nach (i) ist dann $f\left(0_{i+1}\right)$ ausgezeichnet. Wegen Eigenschaft 2 von $f$ ist auch $0_{i+1}$ ausgezeichnet.

In den restlichen Fällen werden die folgenden Objekte und endlichen Teilobjekte betrachtet: 
(i) $=(5)$ abjekte sind unterteilte Graphen mit Komponenten der Grösse $k$ (k endich).

Endliche Teilobjekte sind endliche Teilgraphen bestehend aus endlich vielen Komponenten und den dazwischen liegenden Kanten.

Ausgezeichnet sind Dbjekte mit k-Auswahl-clique.

$\underline{(i)=(6)}$ bbjekte sind Mengen von aussagenlogischen Formeln in kanjunktiver Normalform mit höchstens $k$ Variablen pro Klausel.

Endliche Teilobjekte sind endiche Teilmengen davon.

Ausgezeichnet sind die erfullbaren formelmengen.

(i) $=(1)$ analog $z u(6)$

Nach dem obigen Sohema ist nun auch $(5) \Longrightarrow(6)$ und $(6) \Longrightarrow(1)$ beweisbar. Für den Beweis van (6) $\Longrightarrow$ (1) müssen wir noch eine Transformation angeben, welche einer erfüllbaren (bzw. nicht erfüllbaren) aussagenlogischen Formel F wieder eine erfüllbare (bzw. nicht erfüllbare) Formel $F_{N}$ in konjunktiver Normalform zuordnet.

Dazu wird die Formel $F$ schrittweise van innen her abgebaut, indem z.B. eine Disjunktion von zwei Variablen $x_{a} v x_{b}$ in $F$ ersetzt wird durch eine neue Variable $x_{(a, v, b)}$ während gleichzeitig $F_{N} u m$

$$
\left(\neg \times_{(a, v, b)} \vee \times_{a} \vee x_{b}\right) \wedge\left(\neg x_{a} \vee x_{(a, v, b)}\right) \wedge\left(\neg x_{b} \vee x_{(a, v, b)}\right)
$$

verlängert wird (was bedeutet $x_{(a, v, b)} \Longleftrightarrow\left(x_{a} \vee x_{b}\right)$ ). Nach (III.12) ordnen wir der Formel $F_{N}$ schliesslich eine Formel mit höchstens $k$ Variablen pro Klausel zu.

Damit ist die Aequivalenz der Aussagen (1) bis (6) bewiesen.

\section{Einige direkte Beweise}

Die direkten Transformationen aus dem ersten Kapitel liefern uns jetzt nach dem allgemeinen Schema (i) $\Longrightarrow(i+1)$ sofort direkte Beweise van Implikationen zwischen verschiedenen Aussagen $I, P_{k}$ und $S_{k}$.

Die direkte Transformation von $2^{n}-\left(b z w \cdot n^{2}-\right)$ FAERBBARKEIT auf DREIFAERBBARKEIT (VI.3) Iiefert direkte Beweise von $P_{3} \Longrightarrow P_{2}$ (bzw. $\mathrm{P}_{3} \Longrightarrow \mathrm{P}_{2}$ ). Insbesondere erhalten wir einen direkten Eeweis von $P_{3} \Longrightarrow P_{4}^{n^{2}}$ und lösen damit ein von (Läuchli 1971) gestelltes Problem.

Zur Vermeidung des Auswahlaxioms für die Transformation von unendlichen Graphen müssen wir hier symmetrisieren. Falls $\{p, q\}$ eine Kante von $G$ ist, so werden deswegen in $G$ ' an die Punkte $p_{1} \ldots \ldots p_{n}$ und $q_{1}, \ldots, q_{n}$ zwei Graphen angehängt. Der eine sieht aus wie in der Zeichnung (VI.4), 
der andere ist gespiegelt, so dass das Vieleck näher bei den Punkten $q_{1}, \ldots, q_{n}$ ist. So wird vermieden, dass einer der Punkte $p, q$ ausgezeichnet wird.

Wir beschreiben alle Transformationen immer nur anschaulich, statt sie formal in der Mengenlehre zu definieren. Unsere Beschreibung könnte aber leicht in eine formale Definition übersetzt werden, indem wir z.B. festlegen: $p_{1}$ bezeichnet den Punkt $(p, 1), k_{1}^{2}$ bezeichnet zuerst den Punkt $(p, q, 2,1)$ und nachher $(q, p, 2,1)$ usw.

Die direkte Transformation von $E^{0}$ auf DREIFAERBBARKEIT (VI.5) liefert einen direkten Beweis von $\mathrm{P}_{3} \Longrightarrow I$.

Die direkte Transformation von k-AUSWAHL-CLIQUE auf k- (bzw. 3-)FAERBBARKEIT (VI.7) liefert direkte Beweise von $P_{k} \Longrightarrow s_{k}$ für $k \geq 3$ und $' P_{3} \Longrightarrow s_{k}$ für $k \geq 1$. In der Definition von $P$ ' ersetzen wir $i<j$ durch $i \leq j$.

Die direkte Transformation von $(m \cdot n)$-AUSWAHL-CLIQUE nach $(m+n-1)$-AUSWAHL CLIQUE (VI.g) liefert direkte Beweise von $s_{m+n-1} \Longrightarrow s_{m \cdot n}$, insbesondere $\mathrm{S}_{3} \Longrightarrow \mathrm{S}_{4}$.

Damit wir die Transformation auch für unendliche unterteilte Graphen definieren können (ohne Auswahlaxiom) müssen wir hier wieder symmetrisieren: Wir nehmen nicht nur eine Aufteilung der Komponenten der Grösse $m \cdot n$ in $n$ Komponenten der Grösse $m$, sondern gleich alle $\frac{(m \cdot n):}{m ! n}$ solchen Aufteilungen. So müssen wir keine Reihenfolge der Elemente einer Komponente auszeichnen.

\section{Literatur}

Läuchli, H., Coloring infinite graphs and the Boolean prime ideal theorem, Israel J. Math. 9 (1971) 422-429.

Lévy, A., Remarks on a paper by J. Mycielski, Acta Math. Acad. Sci. Hungar. 14 (1963) 125-130.

toś, J. and Ryll-Nardzewski, C., Effectiveness of the representation theory for Boolean algebras, Fund. Math. 41 (1955) 49-56.

Mycielski, J., Some remarks and problems on the colouring of infinite graphs and the theorem of Kuratowski, Acta Math. Acad. Sci. Hungar. 12 (1961) 125-129. 